Production, measurement and simulation of a low mass flex cable for multi gigabit/s readout for the LHCb VELO upgrade

This content has been downloaded from IOPscience. Please scroll down to see the full text. 2013 JINST 8 C01018

(http://iopscience.iop.org/1748-0221/8/01/C01018)

View the table of contents for this issue, or go to the journal homepage for more

Download details:

IP Address: 137.138.125.163

This content was downloaded on 08/07/2014 at 09:10

Please note that terms and conditions apply. 


\title{
Production, measurement and simulation of a low mass flex cable for multi gigabit/s readout for the LHCb VELO upgrade
}

\author{
E. Lemos Cid, ${ }^{a, c, 1}$ J. Buytaert, ${ }^{b}$ A.A. Gallas Torreira, ${ }^{a}$ D. Esperante Pereira, ${ }^{a}$ \\ P. Arne Ronning, ${ }^{b}$ J. Visniakov, ${ }^{a}$ M.G. Sanchez ${ }^{c}$ and P. Vazquez Regueiro ${ }^{a}$ \\ ${ }^{a}$ Universidad de Santiago de Compostela, \\ Santiago de Compostela, Spain \\ ${ }^{b}$ European Organization for Nuclear Research (CERN), \\ Geneva, Switzerland \\ ${ }^{c}$ Universidade de Vigo, \\ Pontevedra, Spain
}

E-mail: edgarlemoscid@gmail.com

ABSTRACT: The goal of this project is to examine the feasibility of data transmission up to $\sim 5 \mathrm{Gbit} / \mathrm{s}$ on a short $(\sim 60 \mathrm{~cm})$ low mass flex cable, for the readout of the upgraded vertex detector (VELO) of the LHCb experiment. They will be in a vacuum and very high radiation environment and also partly in the particle acceptance. For the full system 1600 readout links will be required. A set of single-ended and differential (edge-coupled) striplines, with a variety of line parameters have been prototyped using a material specifically tailored for this type of application (Dupont Pyralux AP-plus polyimide). To reduce mass, the total thickness of the cable is kept to $0.7 \mathrm{~mm}$. We will present measurements of the characteristic impedance, insertion and return loss, obtained both from time and frequency domain, as well as a comparison with simulations and expectations. Also the effectiveness of grounded guard traces and the use of ground via holes to reduce crosstalk will be reported. From the measurements we were also able to extract the material properties such as the dielectric constant and loss factor up to several GHz. The measurements were done with a Vector Network Analyzer (VNA), TDR/TDT Digital Sampling Oscilloscope, serial PRBS generator and analyzer for eye diagram and CAD tools such as Agilent ADS and ANSYS HFSS simulators.

KEYWORDS: Detector design and construction technologies and materials; Front-end electronics for detector readout; Special cables

\footnotetext{
${ }^{1}$ Corresponding author.
} 


\section{Contents}

1 Introduction 1

2 Cable description 2

3 First prototype measurement 2

3.1 Frequency domain 2

3.2 Time domain 4

4 Second prototype $\quad 6$

5 Conclusions $\quad 7$

\section{Introduction}

For the upgraded vertex detector (VELO) of the LHCb experiment, 1600 multi gigabit/s readout links will be required. The first $60 \mathrm{~cm}$ of these links will be in a vacuum and very high radiation environment and also partly in the particle acceptance.

We design two prototypes to prove that the transmission is possible up to at least $5 \mathrm{GHz}$ with a set of single-ended and differential (edge-coupled) striplines with a variety of line parameters.

With this first prototype, we measured several effects in the frequency domain, such as:

- Guard traces between the striplines.

- Via holes connecting the guard traces to the ground planes.

- Surface mount right angle SMA Connectors [4].

- Physical line parameters: variation of trace width, inter-trace gap and length in addition to single and differential line approaches.

- Micro miniature connectors (MOLEX SlimStack $0.4 \mathrm{~mm}$ ) [3].

And, several effects in time domain:

- The characteristic impedance profile along the line.

- Eye diagrams.

In the second prototype, currently in production we include several enhancements, such as a new SMA launch pad layout, an improved race-track routing resulting in lower intra-pair skew and a reduced dielectric thickness to improve cable flexibility and total mass. 


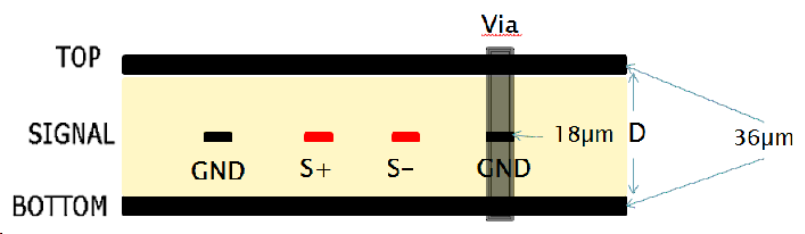

Figure 1. Cable section.

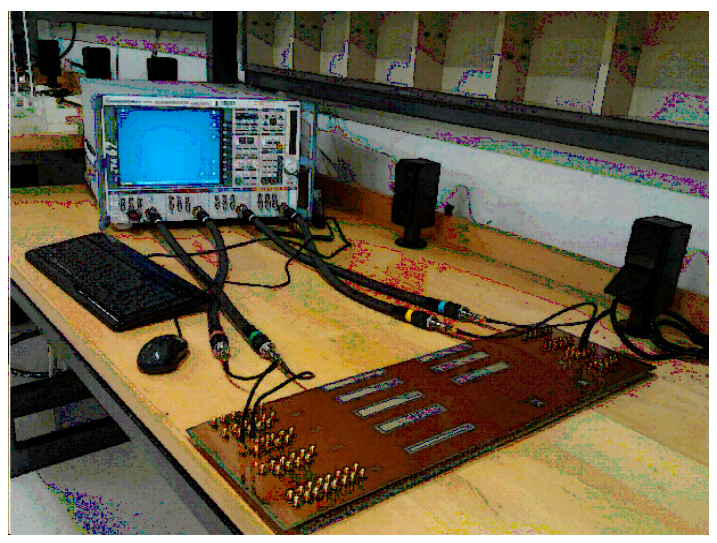

Figure 2. Rohde \& Schwarz ZVA Vector Network [8].

\section{Cable description}

The two prototypes are composed of differential coplanar striplines with isolating guard traces between pairs. The cables are made with Dupont Pyralux AP-plus polyimide [2] with dielectric constant 3.4 and dissipation factor (Loss tangent) of 0.002 at $5 \mathrm{GHz}$.

Figure 1 shows the section of the differential coplanar stripline, with the use of via's on some of the ground traces.

The thickness of the dielectric (D in figure 1) is different in the two prototypes: $\sim 500 \mu \mathrm{m}$ in prototype one and $\sim 350 \mu \mathrm{m}$ in prototype two. We also used a cover lay on top and bottom layer of about $65 \mu \mathrm{m}$ thickness.

\section{First prototype measurement}

\subsection{Frequency domain}

We simulated the first prototype [1] in frequency domain with momentum microwave of ADS of Agilent [5] and ANSYS HFSS [6] and we measured with Rohde\&Schwarz ZVA Vector Network Analyzer 4 ports, $67 \mathrm{GHz}$ (figure 2).

We measured:

- The crosstalk between two differential transmission lines. Guard traces (named GND in figure 1) were added to isolate all the striplines. On some guard traces additional via holes were added every $10 \mathrm{~mm}$ to connect it with the top and bottom GND layers to further reduce 

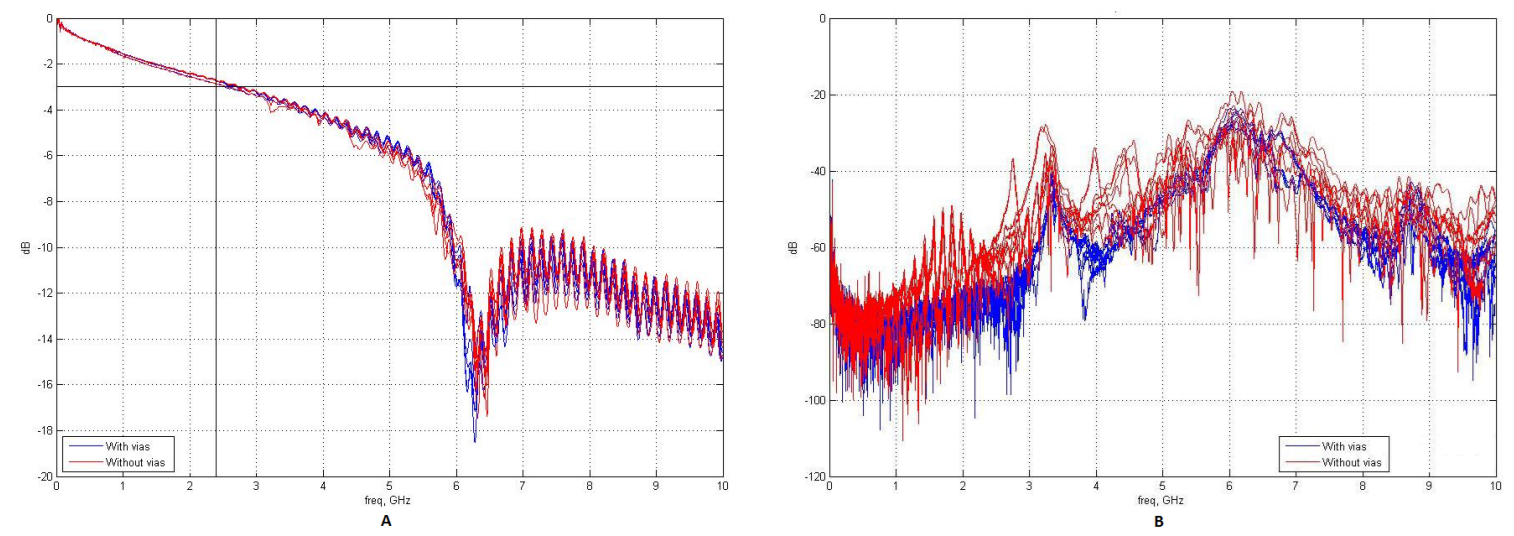

Figure 3. Parameter S21 differential (A) and crosstalk (B) between striplines with and without vias.

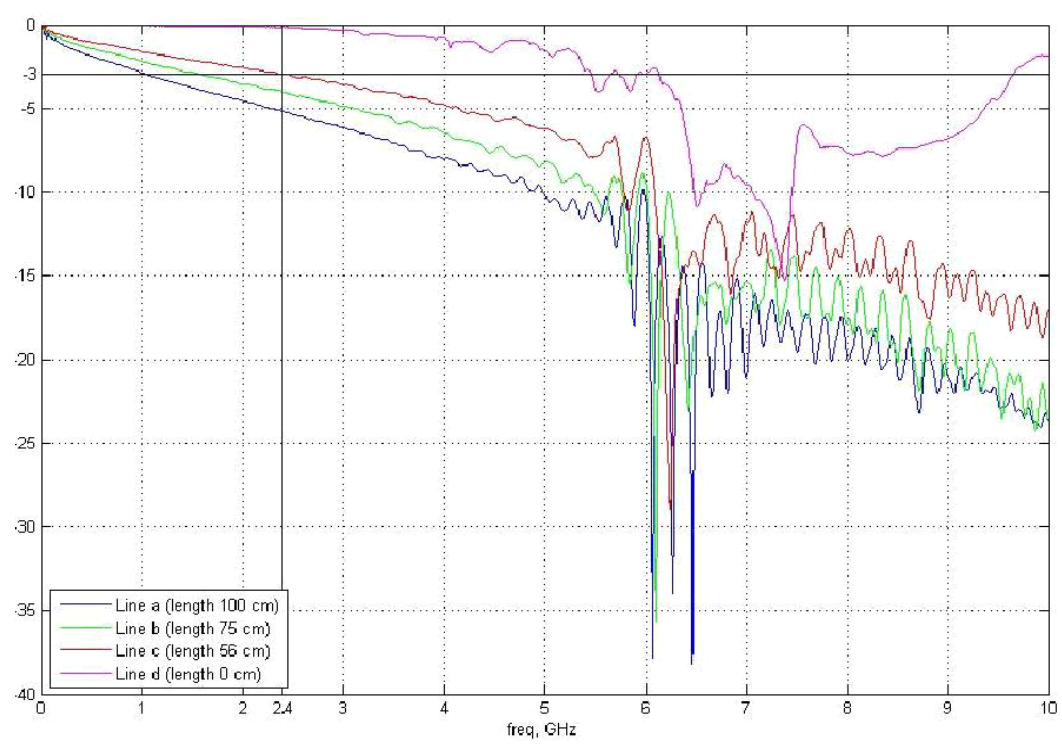

Figure 4. Parameter S21 differential of several length lines.

crosstalk. Figure 3 (A), shows the measured parameter S21 differential [9] of two lines with (red) and without (blue) additional via's. Figure 3 (B), plots the crosstalk between two adjacent striplines with (red) and without (blue) via's. From these measurements we conclude that additional via's on the guard traces do not affect significantly the transmission nor do they contribute to further suppress crosstalk.

- The dependence of the transmission performance (S parameters) on physical line parameters. For this purpose, lines were designed where the trace width varied from 250 to $310 \mu \mathrm{m}$, intertrace gap from 150 to $750 \mu \mathrm{m}$ and length from 50 to $100 \mathrm{~cm}$. Figure 4 shows the parameter S21 differential of lines with different length. As a rule of thumb, for data transmission at $4.8 \mathrm{~Gb} / \mathrm{s}$, the attenuation should not exceed more than $3 \mathrm{db}$ at $2.4 \mathrm{Ghz}$. From figure 4 this seems possible for a line with length less than $56 \mathrm{~cm}$. 


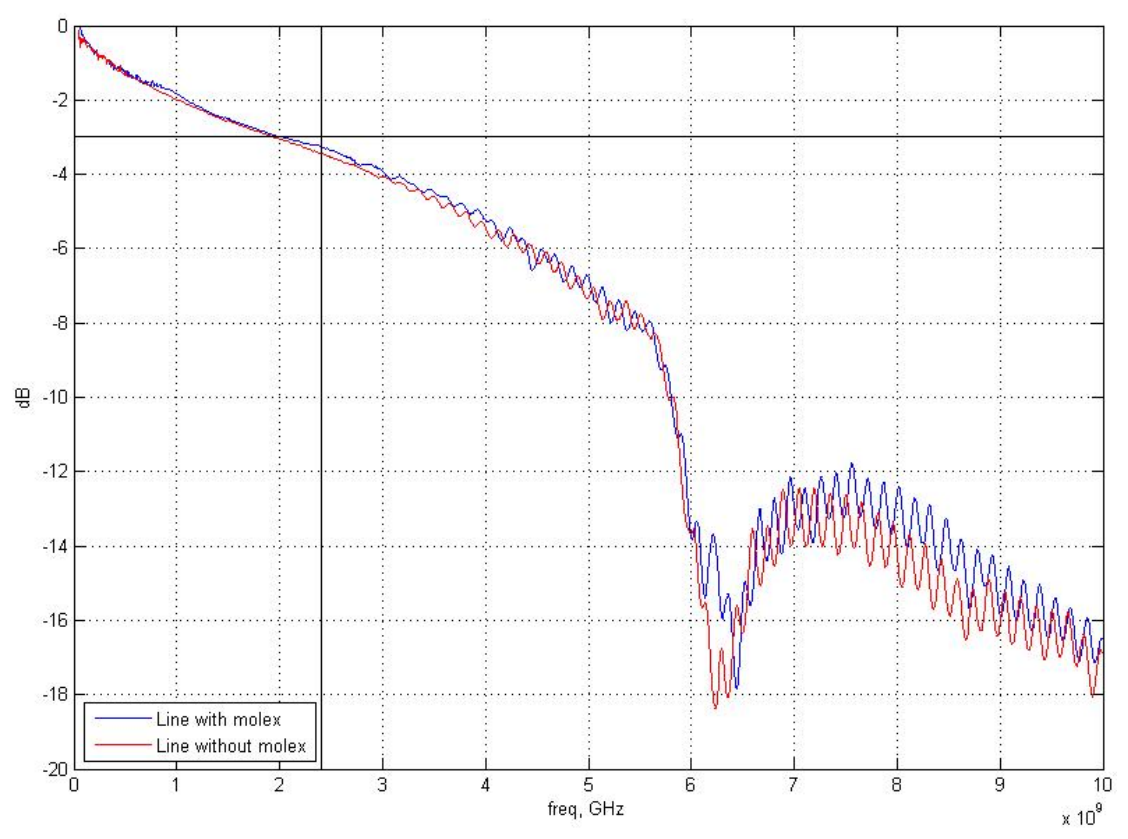

Figure 5. Parameter S21 differential of two striplines with Molex connector and without connector.

- We measured the effect of the use of a micro miniature connector (MOLEX SlimStack $0.4 \mathrm{~mm}$ ). Figure 5 shows the parameter S21 differential of two striplines with and without the connector. The insertion loss is less than $0.1 \mathrm{~dB}$ at $5 \mathrm{Gbps}$.

- Surface mount right angle SMA Connectors were used to connect the in- and outputs of all the lines. To avoid routing via's on the signal traces to go from the top to the middle layer; we implemented a layout that allowed mounting the connectors directly on the middle layer instead of the top layer. Nevertheless a sharp insertion loss appeared around $6 \mathrm{GHz}$ (visible on all the above plots). By using a 3D EM-field simulation (Ansys HFSS) we could explain it as a resonance in a parasitic cavity and also design an improved layout. In figure 6, we plot the e-fields for the SMA with the original and the improved layout, showing clearly that the energy of the signal is contained in the improved layout.

\subsection{Time domain}

In time domain, we measured the characteristic impedances with the Tektronix TDS 8000 Digital Sampling Oscilloscope [7] and the eye diagram with the two instruments: Agilent J-Bert N4903B High-Performance Serial Bert and Agilent Infiniium DSA91204A Digital Signal Analyzer (figure 7).

We measured the following two effects:

- Eye diagrams were measured at 4 and $8 \mathrm{GHz}$. We compare them with simulated eye diagrams obtained with the Lecroy SI-studio software. The eye diagram stays well open at a transmission speed of $4 \mathrm{GHz}$ and $1 \mathrm{~m}$ (figure 8 ). At $8 \mathrm{GHz}$ the eye is nearly closed. 

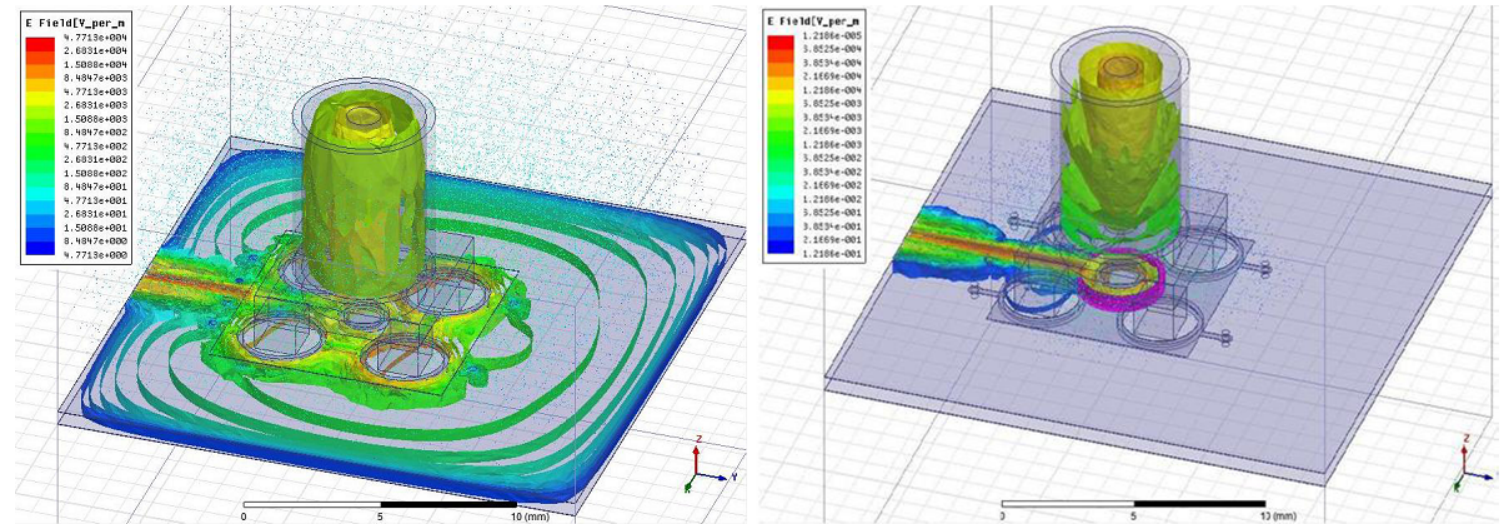

Figure 6. SMA Fields at $6 \mathrm{GHz}$ with the original and the improved layout.
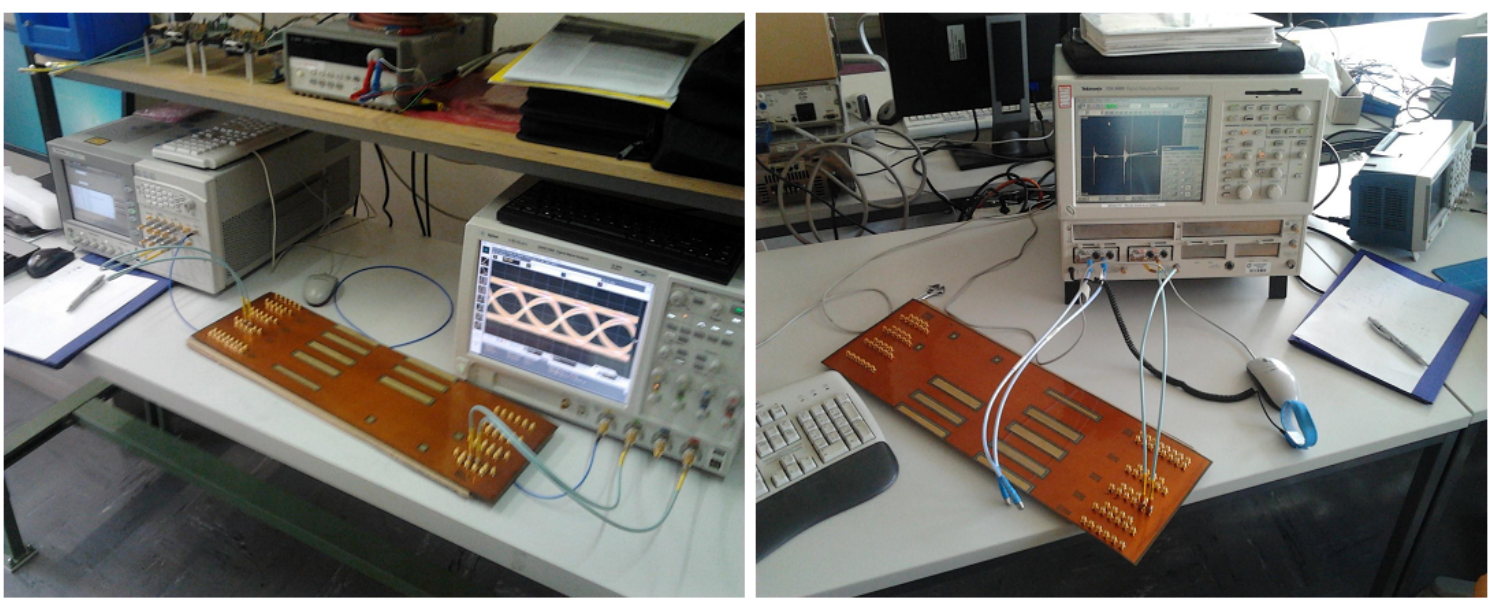

Figure 7. Agilent J-Bert N4903B High-Performance Serial Bert and Agilent Infiniium DSA91204A Digital Signal Analyzer (Left). Tektronix TDS 8000 Digital Sampling Oscilloscope (Right).

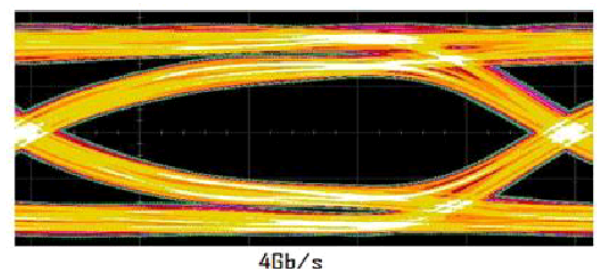

$4 \mathrm{~Gb} / \mathrm{s}$

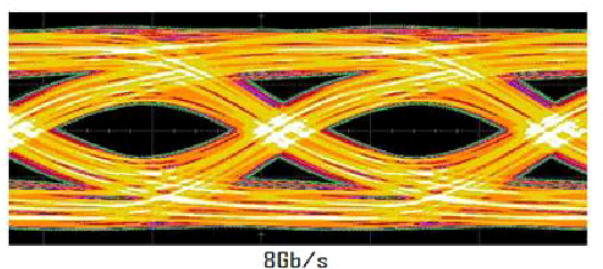

$100 \mathrm{~cm}$

Figure 8. Eye diagrams for a cable of 1 meter for $4 \mathrm{~Gb} / \mathrm{s}$ and $8 \mathrm{~Gb} / \mathrm{s}$.

- The characteristic impedance of the various lines. The values correspond very well with the predicted values obtained with the mwi2010 calculator from Rogers corp. (figure 9). We also plot the impedance profile along a line in figure 10. A clear 'ringing' appears at the transition between the test cable and the measurement cable. The period $(\sim 170 \mathrm{ps})$ of this damped oscillation corresponds very well to the absorption peak in S21 at $6 \mathrm{GHz}$. From the slope of the impedance we could extract the attenuation. 

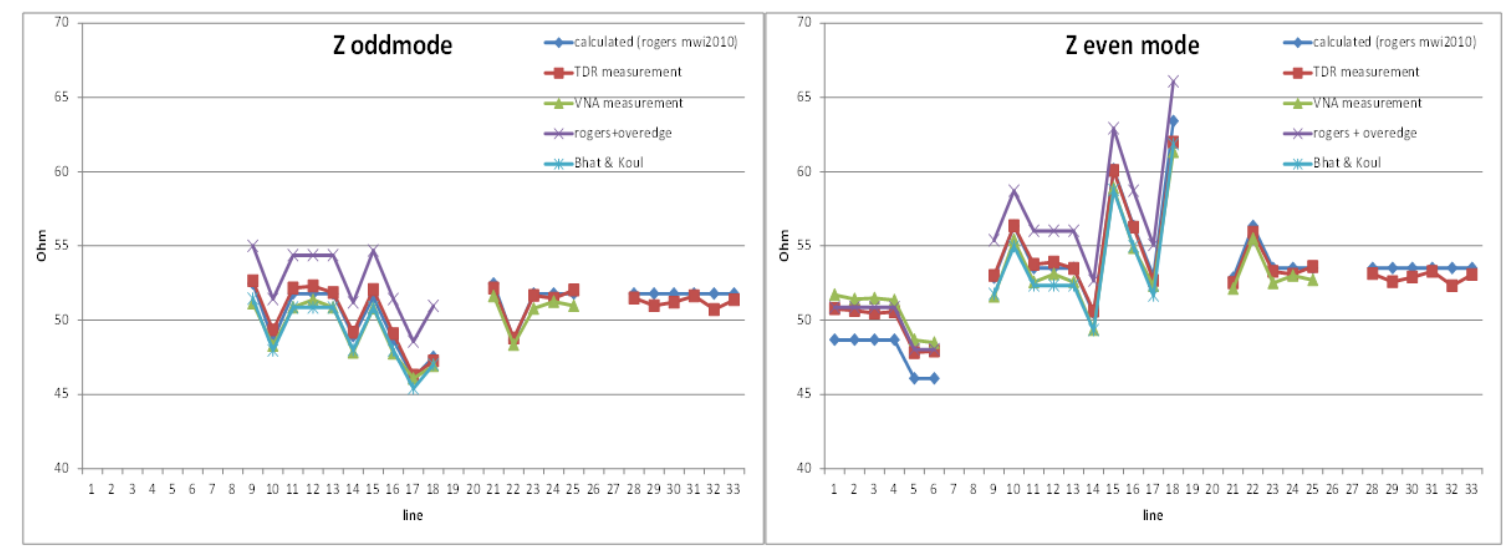

Figure 9. Impedance calculation with the simulator and measurement in odd and even mode [10] for different lines.
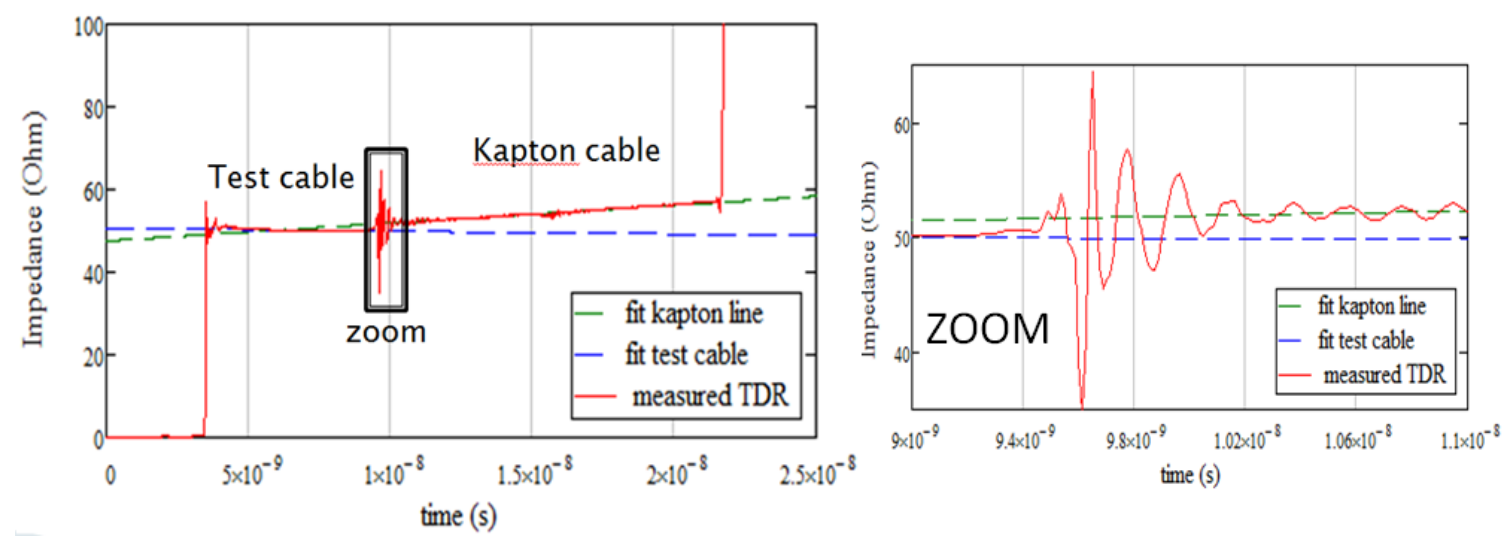

Figure 10. Impedance measurement along the test and measurement (Kapton) cables with zoom of the ringing effect.

\section{Second prototype}

We have designed a second prototype with several enhancements:

- Thinner laminates (350 um of dielectric (D)) and narrower traces (to maintain the same impedance). This will increase the flexibility of the cable.

- Improved SMA pad layout to avoid resonant insertion loss. We included a ring of via's between the top and bottom layers around the SMA signal pin to contain the signal.

- For improved testing, we added several features to be able to eliminate all contribution from test cables and connectors (de-embedding with a 'THRU' calibration technique).

- Equalize left and right turns in signal traces to minimize intra-pair skew.

- Include bonding wires, to study their effect on the transmission performance. 


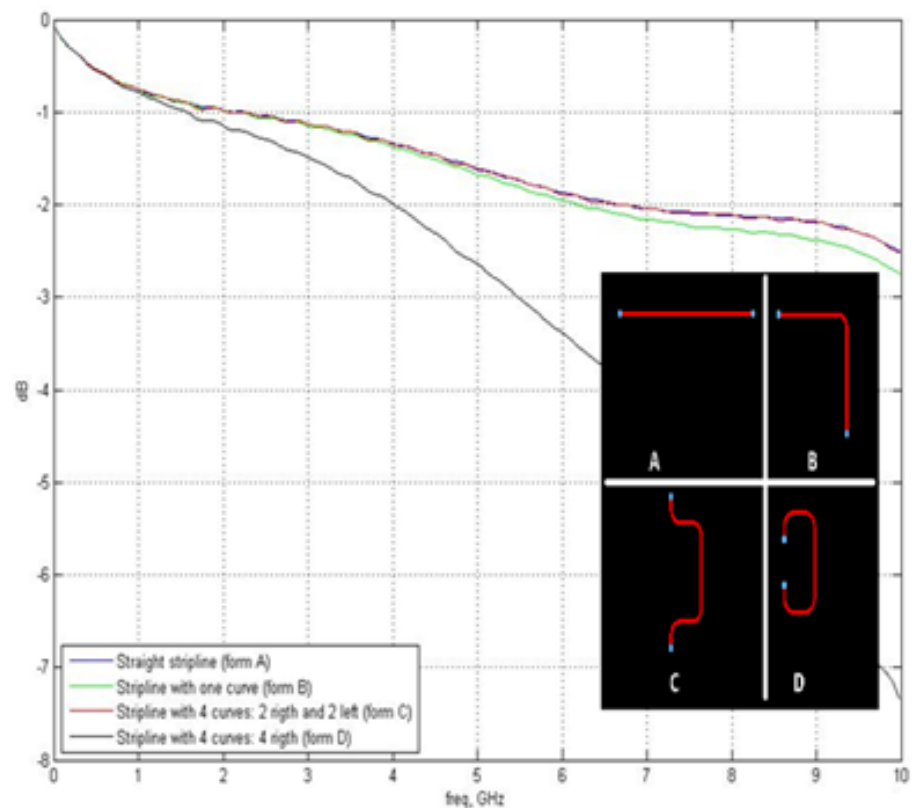

A

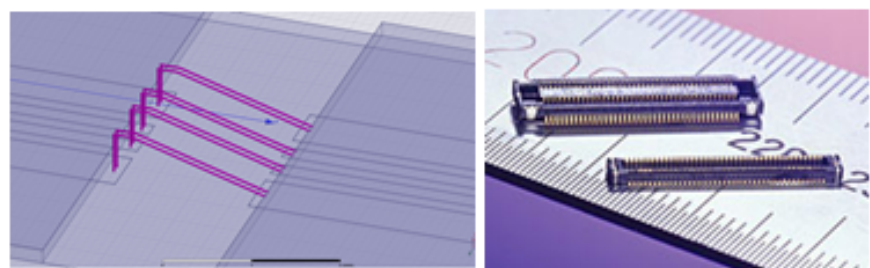

B $\mathrm{C}$

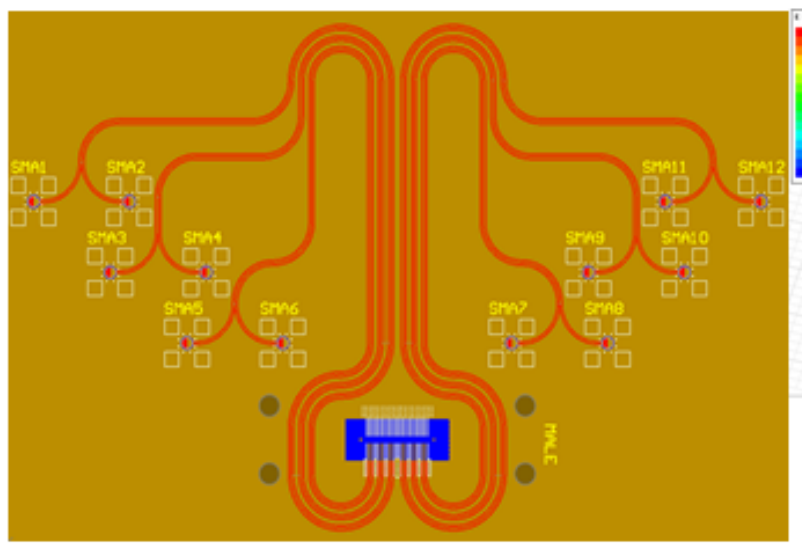

$\mathrm{D}$

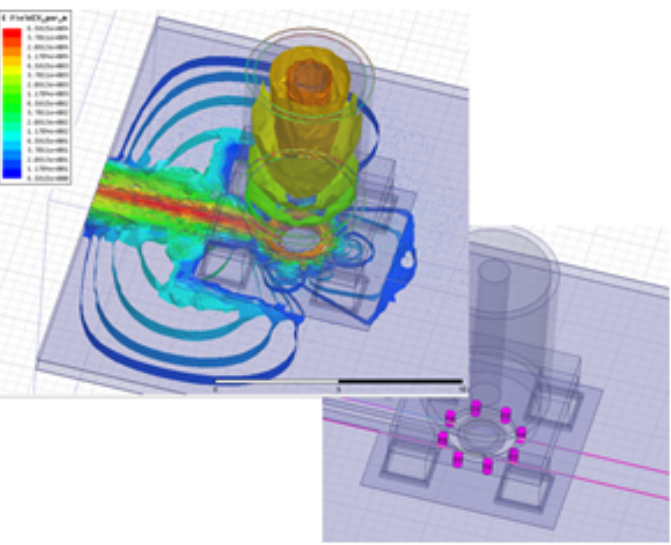

$\mathrm{E}$

Figure 11. Some enhancements: equalize left and right turns in signal traces to minimize inter-pair skew (A), bonding (B), Molex connector (C), SMA-Molex adapter (D), Improve SMA pad layout (E).

\section{Conclusions}

With this first prototype, we showed the possibility to make a low mass cable, with very small interpair crosstalk, for high speed transmission of $5 \mathrm{Gbps}$ over $60 \mathrm{~cm}$. A second prototype, with improved layout and new features is under production. 


\section{References}

[1] J. Buytaert, D. Esperante, P. Vázquez and J. Visniakov, Design and test of a prototype of a flex cable for high speed transmission, presented on the regular VELO Upgrade discussion meeting on 24.6.2011: http://fpmac116.usc.es/twiki/pub/Instrumentation/VeloUpgradeCable/ VeloUpgradeCablePablo.pptx.

[2] Pyralux AP-Plus datasheet: http://www2.dupont.com/Pyralux/en_US/assets/downloads/pdf/ Pyralux_AP-Plus_DataSheet.pdf.

[3] Molex connector: http://www.molex.com/molex/products/datasheet.jsp?part=active/ 5024304410_PCB_HEADERS.xml.

[4] SMA connector datasheet: https://emersonconnectivity.com/OA_MEDIA/specs/pi-142-0711-201.pdf.

[5] ADS of Agilent web page: http://www.home.agilent.com/agilent/product.jspx?cc=ES\&lc=eng \&ckey=1297113\&nid=-34346.0.00\&id=1297113.

[6] ANSYS HFSS web page: http://www.ansys.com/Products/Simulation+Technology/Electromagnetics/ High-Performance+Electronic+Design/ANSYS+HFSS.

[7] Tektronix TDS 8000 Digital Sampling Oscilloscope manual: http:/www.ko4bb.com/Manuals/ Tektronix/Tektronix_-_CSA8000_Communication_System_Analyzer/CSA8000-User-071043302.pdf.

[8] Rohde \& Schwarz ZVA Vector Network Analyzer 4 ports, $67 \mathrm{GHz}$ manual: http://www2.rohde-schwarz.com/file/ZVA_ZVB_ZVT_Operating.pdf.

[9] W. Fan, A.C. W. Lu, L.L. Wai and B.K. Lok, Mixed-Mode S-Parameter Characterization of Differential Structures, in proceedings of Electronic Packaging Technology Conference (2003) 533.

[10] Even and Odd mode impedance - an introduction, http://www.polarinstruments.com/support/cits/ AP157.html. 